\title{
Four-dimensional transverse beam matrix measurement using the multiple-quadrupole scan technique
}

\author{
Eduard Prat* and Masamitsu Aiba \\ Paul Scherrer Institut, CH-5232 Villigen PSI, Switzerland \\ (Received 10 July 2013; published 19 May 2014)
}

\begin{abstract}
Accurate measurements of the transverse beam properties are essential to understand and optimize particle beams. We present an optimized method that uses three quadrupole magnets and one profile monitor to measure the full 4D transverse matrix of the beam. The method has been applied to the SwissFEL Injector Test Facility (SITF) at the Paul Scherrer Institute (Villigen). The SITF is the principal test bed and demonstration plant for the SwissFEL project, which aims at realizing a hard-x-ray freeelectron laser in 2017. Simulations, measurements, and results of cross-plane coupling correction are presented.
\end{abstract}

DOI: 10.1103/PhysRevSTAB.17.052801

PACS numbers: 29.20.Ej, 41.60.Cr, 41.85.-p, 29.27.Fh

\section{INTRODUCTION}

Precise knowledge of the transverse beam parameters is essential in all types of charged particle accelerators. In this study we focus on the transverse properties of the electron beam in free-electron lasers (FEL), where a high quality beam with low emittance and matched optics is required for the lasing process.

Procedures to measure the two-dimensional (2D) transverse beam parameters, where the horizontal and vertical motions are treated separately, have been well established - see, for instance, Refs. [1-7]. However, various sources such as tilted quadrupole magnets, sextupole misalignments, or solenoid field asymmetries generate a correlation between the horizontal and vertical motions. Moreover, the initial beam from an electron gun may have a strong crossplane coupling. These effects will result in an increase of the apparent 2D emittances, i.e., the emittances projected onto the horizontal and vertical planes. For FELs this would imply a deterioration of the laser performance. A full fourdimensional (4D) measurement becomes necessary to measure precisely and possibly correct the transverse coupling.

Woodley and Emma [8] proposed to measure the full 4D matrix by using a long beam line of $\sim 150 \mathrm{~m}$ consisting of six profile monitors and $\sim 15$ quadrupole magnets. Their design was based on the single-optics/multiple-locations strategy: the beam parameters were measured at the six profile monitors with a fixed optics along the lattice.

We present a method to measure the 4D beam parameters based on the multiple-optics/single-location approach: the

"eduard.prat@psi.ch

Published by the American Physical Society under the terms of the Creative Commons Attribution 3.0 License. Further distribution of this work must maintain attribution to the author $(s)$ and the published article's title, journal citation, and DOI. beam parameters (beam sizes and coupling correlation) are measured at a single position and quadrupole strengths are changed to generate the required optics for the 4D measurement. Four-dimensional measurements by means of quadrupole scans have already been reported in the past $[9,10]$. Here we propose using the multiple-quadrupole scan technique to optimize the optics for the 4D measurement, which results in a minimization of the error of the reconstructed parameters.

The multiple-optics/single-location approach is more efficient since it requires much less space and components - our method utilizes a compact setup of about $10 \mathrm{~m}$ with only three quadrupoles and one profile monitor.

The correction of the beam coupling can be achieved by skew quadrupoles and/or solenoids. While in Ref. [10] the correction of a single coupling term by using one skew quadrupole only seemed to be sufficient, a more complete correction scheme should be envisaged in general. The strategy presented in Ref. [11] for the Linac Coherent Light Source (LCLS) at SLAC cannot be applied in general either because it is based on an empirical optimization feasible in the case where only a few parameters are involved. We implemented a more general tool allowing the correction of all coupling terms.

In Sec. II, the 4D beam characterization is reviewed and formulated. A systematic comparison between the singleoptics/multiple-locations and the multiple-optics/singlelocation strategies as well as our measurement procedure are discussed in Sec. III. An application at the SwissFEL Injector Test Facility (SITF) [12], including simulations, measurements, and correction, is presented in Sec. IV. Finally, the conclusions are drawn in Sec. V.

\section{4D TRANSVERSE BEAM CHARACTERIZATION AND TRANSPORT}

The 4D beam matrix describes the transverse statistical properties of the beam: 


$$
\sigma^{4 D}=\left(\begin{array}{cccc}
\left\langle x^{2}\right\rangle & \left\langle x x^{\prime}\right\rangle & \langle x y\rangle & \left\langle x y^{\prime}\right\rangle \\
\left\langle x x^{\prime}\right\rangle & \left\langle x^{\prime 2}\right\rangle & \left\langle x^{\prime} y\right\rangle & \left\langle x^{\prime} y^{\prime}\right\rangle \\
\langle x y\rangle & \left\langle x^{\prime} y\right\rangle & \left\langle y^{2}\right\rangle & \left\langle y y^{\prime}\right\rangle \\
\left\langle x y^{\prime}\right\rangle & \left\langle x^{\prime} y^{\prime}\right\rangle & \left\langle y y^{\prime}\right\rangle & \left\langle y^{\prime 2}\right\rangle
\end{array}\right)=\left(\begin{array}{cc}
\sigma_{x x} & \sigma_{x y} \\
\sigma_{x y}^{T} & \sigma_{y y}
\end{array}\right),
$$

where $x$ and $y$ are the horizontal and vertical coordinates, respectively, and $x^{\prime}$ and $y^{\prime}$ are their derivatives with respect to the longitudinal coordinate. The matrices $\sigma_{x x}$ and $\sigma_{y y}$ describe the 2D horizontal and vertical motions, and $\sigma_{x y}$ describes the cross-plane coupling. If the beam is transversely decoupled $\left(\sigma_{x y}=0\right)$ the $2 \mathrm{D}$ beam matrices are sufficient to characterize the beam. The apparent emittances and the so-called Twiss parameters can be obtained from the $2 \mathrm{D}$ matrices as follows:

$$
\begin{gathered}
\epsilon_{u}=\sqrt{\operatorname{det} \sigma_{u u}}, \\
\beta_{u}=\left\langle u^{2}\right\rangle / \epsilon_{u}, \quad \gamma_{u}=\left\langle u^{\prime 2}\right\rangle / \epsilon_{u}, \quad \alpha_{u}=-\left\langle u u^{\prime}\right\rangle / \epsilon_{u},
\end{gathered}
$$

where $u$ refers to either $x$ or $y$. When we multiply the emittance by the total momentum we get the so-called normalized emittance:

$$
\epsilon_{N u}=\frac{p}{m c} \epsilon_{u},
$$

where $p$ is the central momentum of the beam, $m$ is the particle rest mass, and $c$ is the speed of light.

If one or more elements of $\sigma_{x y}$ are nonzero, the beam is $x-y$ coupled and the projections onto the $x$ and $y$ planes exhibit an increase of the observed emittance values. The diagonalization of the 4D beam matrix yields the intrinsic emittances $\epsilon_{1}$ and $\epsilon_{2}$ :

$$
U^{T} \sigma^{4 D} U=\left(\begin{array}{cccc}
\epsilon_{1} & 0 & 0 & 0 \\
0 & \epsilon_{1} & 0 & 0 \\
0 & 0 & \epsilon_{2} & 0 \\
0 & 0 & 0 & \epsilon_{2}
\end{array}\right) .
$$

It can be proved that the product of the apparent emittances cannot be smaller than the product of the intrinsic emittances [13]:

$$
\epsilon_{1} \epsilon_{2} \leq \epsilon_{x} \epsilon_{y} .
$$

The transverse coupling contribution to the apparent emittances can be quantified by the coupling factor,

$$
C=\sqrt{\frac{\epsilon_{x} \epsilon_{y}}{\epsilon_{1} \epsilon_{2}}}-1 \geq 0 .
$$

For $C=0$ the beam is decoupled and the apparent emittances have minimal values, while for $C>0$ the beam is transversely coupled and the apparent emittances are increased.
The transport of the 4D beam matrix from $s_{0}$ to $s$ can be calculated as follows:

$$
\sigma_{s}^{4 D}=R \sigma_{s_{0}}^{4 D} R^{T},
$$

where $R$ is the transport matrix between $s_{0}$ and $s$ :

$$
R=\left(\begin{array}{llll}
R_{11} & R_{12} & R_{13} & R_{14} \\
R_{21} & R_{22} & R_{23} & R_{24} \\
R_{31} & R_{32} & R_{33} & R_{34} \\
R_{41} & R_{42} & R_{43} & R_{44}
\end{array}\right)=\left(\begin{array}{ll}
R_{x x} & R_{x y} \\
R_{y x} & R_{y y}
\end{array}\right) .
$$

The lattice is coupled if any of the elements of $R_{x y}$ or $R_{y x}$ are nonzero. The degree of coupling in the final beam at $s$ depends on the initial beam coupling and the lattice properties between $s_{0}$ and $s$.

\section{RECONSTRUCTION OF THE 4D PARAMETERS}

According to Eqs. (1), (8), and (9), when the lattice between $s_{0}$ and $s$ does not contain sources of transverse coupling, i.e., the elements of $R_{x y}$ and $R_{y x}$ vanish, the beam sizes and the $x-y$ correlation at $s$ can be expressed in terms of the $\sigma^{4 D}$ elements as follows:

$$
\begin{aligned}
\left\langle x^{2}\right\rangle_{s}= & R_{11}^{2}\left\langle x^{2}\right\rangle_{s_{0}}+R_{12}^{2}\left\langle x^{\prime 2}\right\rangle_{s_{0}}+2 R_{11} R_{12}\left\langle x x^{\prime}\right\rangle_{s_{0}}, \\
\left\langle y^{2}\right\rangle_{s}= & R_{33}^{2}\left\langle y^{2}\right\rangle_{s_{0}}+R_{34}^{2}\left\langle y^{\prime 2}\right\rangle_{s_{0}}+2 R_{33} R_{34}\left\langle y y^{\prime}\right\rangle_{s_{0}}, \\
\langle x y\rangle_{s}= & R_{11} R_{33}\langle x y\rangle_{s_{0}}+R_{12} R_{33}\left\langle x^{\prime} y\right\rangle_{s_{0}} \\
& +R_{11} R_{34}\left\langle x y^{\prime}\right\rangle_{s_{0}}+R_{12} R_{34}\left\langle x^{\prime} y^{\prime}\right\rangle_{s_{0}} .
\end{aligned}
$$

Therefore assuming the transport matrix elements $R_{i j}$ known, the 10 independent elements of $\sigma^{4 D}$ at $s_{0}$ can be computed by measuring beam sizes and $x-y$ correlations at the point $s$. At least four measurements (i.e., 12 equations) are needed, although more measurements will constrain the problem more tightly. The goal is to find a solution for the beam matrix elements which best fits all the measured data in a least-squares sense. Once $\sigma^{4 D}$ is reconstructed, we obtain the intrinsic emittances and the coupling factor following the procedure described in Ref. [14].

The reconstruction of the 4D transverse beam matrix can be done using two general approaches:

1. Single-optics/multiple-locations. The beam sizes and the $x-y$ correlations are measured at different longitudinal locations while the optics along the lattice is fixed. This measurement strategy was proposed in Ref. [8].

2. Multiple-optics/single-location. The beam dimensions are measured at a single position while the optics between the measurement point and the position where the beam matrix is computed is varied by using one or more quadrupole magnets (see, for instance, Refs. [9] and 
[10]). The advantage with respect to Refs. [9] and [10] of using more quadrupoles at a time, as described in this paper, is the possibility of setting simultaneously horizontal and vertical phase advances, as required by Eq. (12), between point of reconstruction and point of measurement to the desired values, keeping the value of the $\beta$ functions at the point of measurement under control.

The two strategies differ in the following points:

(i) Equipment: The single-optics/multiple-locations approach requires several beam profile monitors while the multiple-optics/single-location approach needs only one. The multiple-optics/single-location scheme requires generally much shorter beam line and less quadrupole magnets, and can be implemented at almost any place of the accelerator. The beam line lengths are typically of the order of $100 \mathrm{~m}$ for the single-optics/multiple-locations approach and of the order of $10 \mathrm{~m}$ for the multiple-optics/singlelocation strategy. In the single-optics/multiple-locations setup, the beam line can be shortened at the expense of higher quadrupole strengths and smaller $\beta$ functions at the measurement position.

(ii) Optics: In the multiple-optics/single-location setup, the covered phase advances and the $\beta$ functions at the measurement position can be chosen freely if multiple quadrupoles are used, while in the single-optics/multiplelocations strategy the optics is fixed by the number of elements and the space between them.

(iii) Errors: The profile monitor may have systematic errors (e.g., calibration and $x-y$ rotation). If they are randomly distributed over the monitors they are more likely to compensate each other in the single-optics/ multiple-locations approach, while such systematic errors will be directly propagated in the multiple-optics/singlelocation setup. In the single-optics/multiple-locations approach the energy error does not necessarily propagate to the emittance error: assuming a matched optics in a FODO (Focus-Drift-Defocus-Drift) cell, the measured normalized emittance is independent of energy measurement errors [15]. The quadrupole field error will have similar impact in both schemes. The small $\beta$ function in a compact lattice in the single-optics/multiple-locations strategy may result in larger beam size measurement errors for a given monitor resolution. Smaller phase-advance steps, easily achievable in the multiple-optics/single-location scheme, may lead to better systematic errors in cases where the transverse beam distribution does not have an ideal elliptic shape in phase space. The two approaches are equivalent in terms of statistical errors.

(iv) Interference with beam operation: In the singleoptics/multiple-locations approach the optics are not modified during the measurement. Therefore this strategy is in principle nondestructive and allows for parasitic measurements (if nondestructive diagnostics like wire scanners are used as profile monitors), while this is not possible in the multiple-optics/single-location setup.
In conclusion, in our case the multiple-optics/singlelocation setup is preferable since it is more flexible, requires less components, and is typically more compact-a beam line of the order of $100 \mathrm{~m}$ adds significant cost to a facility. The systematic error related to the profile monitor can be minimized by a careful monitor setup and a precise beam momentum measurement. The single-optics/multiple-locations setup, however, may be preferred if there is a high demand on less invasiveness to beam operation.

In our method three quadrupoles are used to control the phase advance in one plane and the $\beta$ function at the measurement location in both planes. In the first half of the scan the horizontal phase advance is scanned progressively while the vertical phase advance is kept close to a constant value. In the second part the actions on the horizontal and vertical phase advances are swapped. The horizontal and vertical 2D beam parameters are reconstructed from the first and the second half of the scan, respectively, and the coupling terms are obtained by using the transformations of the full scan.

We note that apart from the two discussed approaches, there are other strategies which can be used to reconstruct the 4D beam parameters, like a multiple-optics/multiplelocations setup. In this study we consider that the quadrupole magnets are placed at a fixed position, but in general the location of a quadrupole can also be tuned-this possibility would circumvent the limitation of beam optics tuning which is attainable by a quadrupole magnet at a fixed location. These and other potentially interesting alternatives are outside the scope of this paper.

\section{APPLICATION AT THE SWISSFEL INJECTOR TEST FACILITY (SITF)}

The SwissFEL project [16], planned at the Paul Scherrer Institute, foresees for 2017 the realization of an x-ray FEL with a radiation wavelength down to $0.1 \mathrm{~nm}$. The SITF [12] is a $250 \mathrm{MeV}$ accelerator with the primary goal of demonstrating the high-brightness electron beam required to drive SwissFEL. The SITF also serves as a test bed for the development and validation of accelerator components needed for the SwissFEL project.

\section{A. Measurement setup and optics}

Figure 1 shows a schematic view of the SITF setup for measuring the electron beam transverse parameters at the

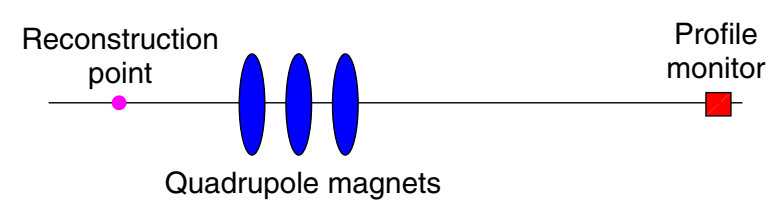

FIG. 1. Sketch of the measurement setup (not to scale). 


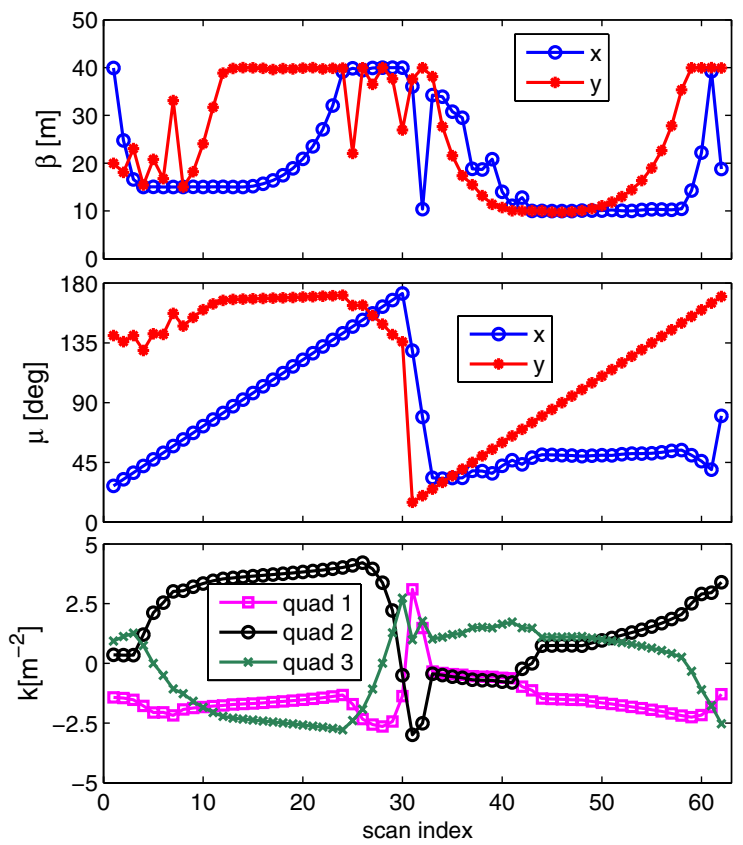

FIG. 2. $\quad \beta$ functions at the observation point, phase advances and quadrupole strengths as a function of the scan index. The first half of the scan is used to reconstruct the horizontal parameters, the second part provides the vertical 2D parameters, and the whole scan is used to obtain the coupling terms.

exit of the magnetic bunch compressor. The layout consists of three quadrupoles with a length of $0.15 \mathrm{~m}$ separated by $1.0 \mathrm{~m}$ and $1.2 \mathrm{~m}$ and a profile monitor $10.7 \mathrm{~m}$ downstream of the last quadrupole.
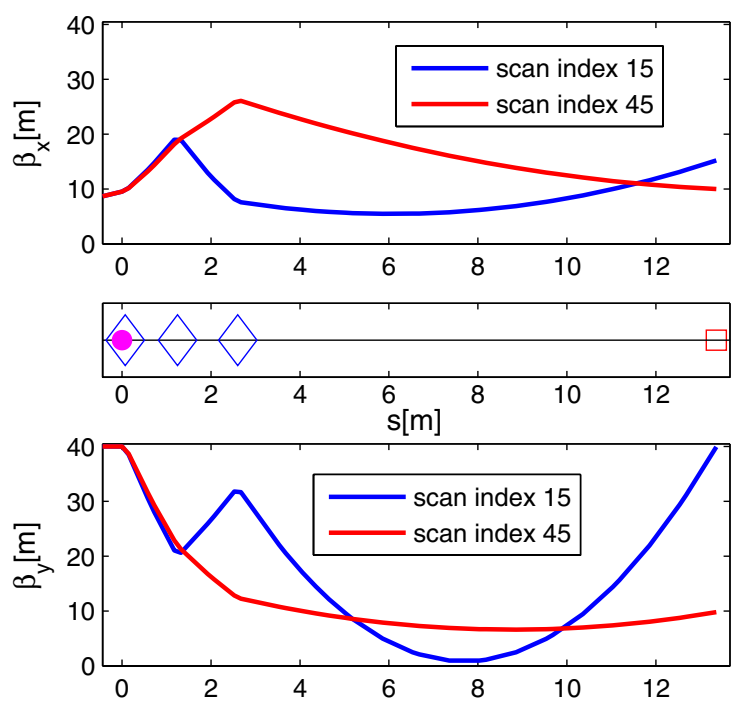

FIG. 3. $\beta$ functions along the measurement lattice for two selected cases (see Fig. 2). The center plot shows the position of the quadrupoles (blue diamonds), the reconstruction point (magenta dot), and the measurement location (red rectangle). The design Twiss values at the reconstruction point are the following: $\beta_{x}=9.51 \mathrm{~m}, \alpha_{x}=-1.03, \beta_{y}=40.02 \mathrm{~m}, \alpha_{y}=-0.02$.
The quadrupole strengths, and the corresponding optics, needed to perform the 4D measurement are calculated by using the code ELEGANT [17]. The phase advances in $x$ and $y$ are regularly scanned in steps of $5^{\circ}$ to cover a total of about $150^{\circ}$. The $\beta$ function at the measurement location is kept between 10 and $40 \mathrm{~m}$, resulting in beam sizes that can be conveniently measured during the whole scan. The quadrupole strengths are constrained to the range $\pm 5 \mathrm{~m}^{-2}$ to limit possible chromatic and dispersive effects. Figure 2 displays the $\beta$ functions at the measurement point, the covered phase advances between the reconstruction point and the measurement location, and the quadrupole strengths as a function of the scan index. Figure 3 shows the $\beta$ function along the measurement lattice for two selected scan indices.
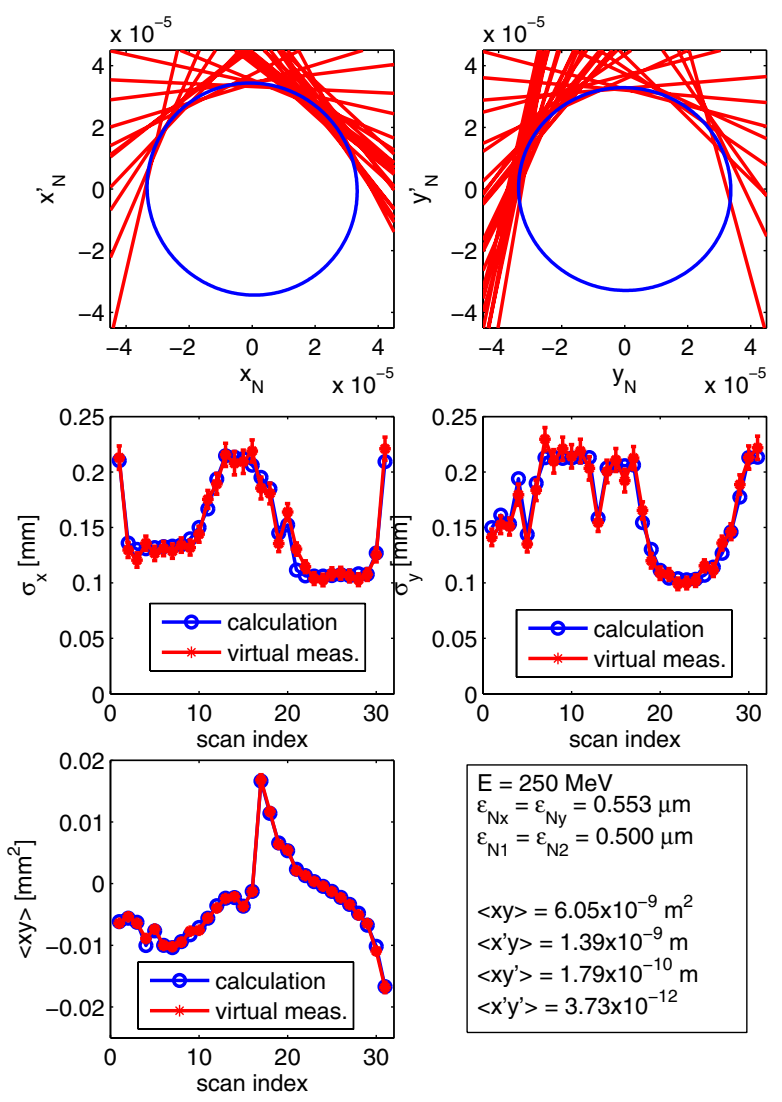

FIG. 4. Simulated measurement example. The nominal beam parameters are indicated in the box. Upper plots show the normalized phase space at the reconstruction point for $x$ (left) and $y$ (right) - the blue circles are the calculated normalized ellipses and the red lines correspond to the beam size measurements transported from the measurement location to the reconstruction point. (The variables $u$ and $u^{\prime}$ are normalized to the measured values: $u_{N}=u / \sqrt{\beta_{u}}, u_{N}^{\prime}=\left(u \alpha_{u}+u^{\prime} \beta_{u}\right) / \sqrt{\beta_{u}}$. In this way the reconstructed ellipses are always circles.) Middle and lower plots show the computed and virtually measured beam sizes and $x-y$ correlations at the measurement location as a function of the scan index. The computed beam sizes are obtained by transporting the calculated beam parameters at the reconstruction point to the measurement location. See also Fig. 5. 

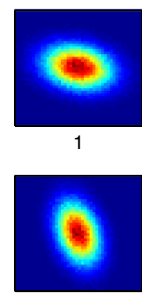

9
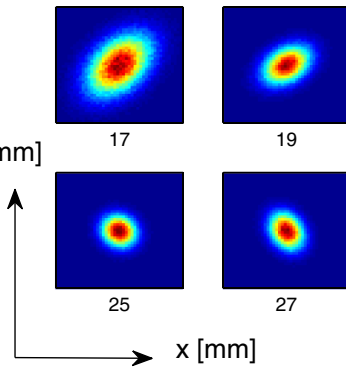

$\mathrm{y}[\mathrm{mm}]$

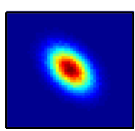

5

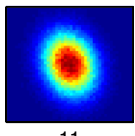

11

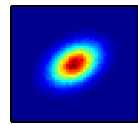

19

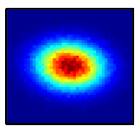

13

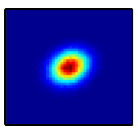

21
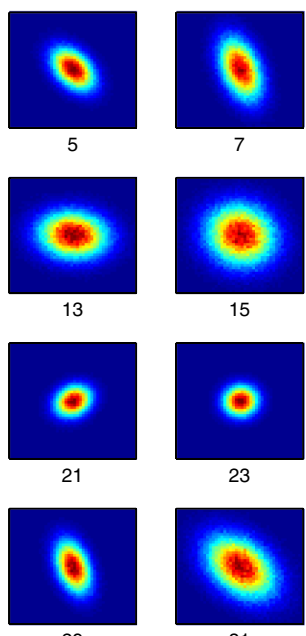

29
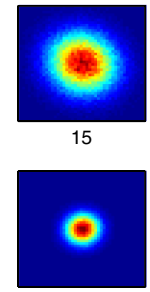

23

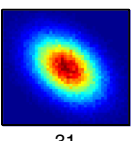

31
FIG. 5. Simulated measurement example: beam images for every other scan step. See also Fig. 4.

We note that the phase advances and $\beta$ functions described in this subsection refer to the lattice and that they are directly related to the transfer matrix elements used in Eqs. (10)-(12) to reconstruct the 4D parameters.

\section{B. Simulations}

The measurement described above has been simulated taking $10^{\circ}$ phase-advance steps for assessing the feasibility of the method and evaluating the effect of errors. Figures 4 and 5 show an example of a simulated measurement, where the apparent emittances increase by $10.7 \%(C=0.107)$ due to coupling. The beam energy is $250 \mathrm{MeV}$, the normalized apparent emittances are $0.553 \mu \mathrm{m}$ in both planes, the optics at the reconstruction point is the nominal one, and the normalized intrinsic emittances are $0.5 \mu \mathrm{m}$ in both planes.

The contributions of statistical and most important systematic errors in the reconstruction of the 4D beam parameters have been estimated for this example as follows:

(i) For evaluating the statistical errors of the reconstructed parameters, simulations with 50000 different seeds were performed. Assuming a relative beam size and $x-y$ correlation measurement error of $5 \%$, the statistical errors of the apparent and the intrinsic emittances are around $2.2 \%$ and $3.0 \%$, respectively.

(ii) The profile monitor resolution limits the emittance measurement resolution and causes a systematic error of the reconstructed emittances. The profile monitor used for the measurements is a Yttrium-aluminum-garnet (YAG) screen with a measured resolution of about $15 \mu \mathrm{m}$. Our simulations show that this corresponds to a normalized emittance resolution of about $0.004 \mu \mathrm{m}$ for an energy of $250 \mathrm{MeV}$. The induced systematic error is around $1.1 \%$ in the $2 \mathrm{D}$ emittances and about $1.7 \%$ in the intrinsic emittances.
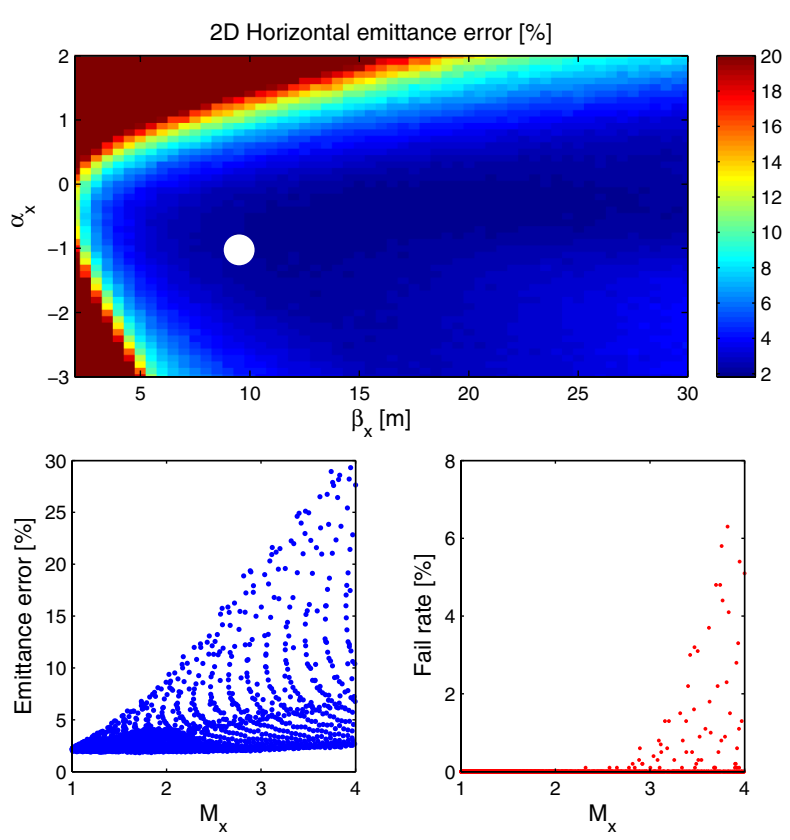

FIG. 6. The upper plot shows the relative horizontal 2D emittance error for different incoming optics. The color code is limited to $20 \%$ to make the details around the matched solution (white dot) visible. A relative beam size measurement error of 5\% is assumed. The lower plots show the emittance error (left) and the failed fits (right) as a function of the mismatch parameter for the cases for which $M \leq 4$. For each point we performed simulations with 1000 different random seeds.

(iii) The calibration error of the profile monitor is of the order of $1 \%$ [18], which causes a systematic error of the reconstructed 2D emittances of around 2\%. ${ }^{1}$ Monte Carlo simulations with 10000 seeds show that this $1 \%$ calibration error translates into a systematic error of the intrinsic emittances of about $2.9 \%$.

(iv) The cross-plane rotation of the profile monitor is of the order of $0.5^{\circ}$ [18]. Our simulation shows that this would result in systematic errors of the intrinsic emittances of $2.7 \%$ and $-0.6 \%$.

(v) Other systematic errors related to the beam size measurement are signal saturation of the profile monitor and beam jitters or drifts. Our YAG screen is equipped with two filters to avoid signal saturation that cut $1 \%$ and $10 \%$ of the total intensity. Concerning the beam jitters, we use single-shot images for the beam size measurement such that the shot-to-shot jitters are avoided. The beam size variation due to machine drifts within a measurement time (less than $2 \mathrm{~min}$ ) is generally not significant enough to disturb the measurement.

(vi) Quadrupole field uncertainties will turn into errors of the transfer matrix elements used to reconstruct the 4D parameters. Magnetic field measurements done at the SITF

${ }^{1}$ Considering that $\sigma=\sqrt{\beta \epsilon}$, the relative error on the emittance evaluation is approximately twice the beam size relative error. 
TABLE I. Summary of systematic errors for the 4D measurement at the SITF.

\begin{tabular}{|c|c|c|}
\hline Source & Effect & Assumption/estimation \\
\hline Profile monitor resolution & $\begin{array}{l}\text { Error in beam parameter } \\
\quad \text { measurements (sizes and }\langle x y\rangle)\end{array}$ & $\begin{array}{l}15 \mu \mathrm{m} \text { in beam size resolution } \\
4 \mathrm{~nm} \text { in emittance resolution } \\
1.1 \% \text { in } 2 \mathrm{D} \text { emittance error } \\
1.7 \% \text { in intrinsic emittance error }\end{array}$ \\
\hline Profile monitor calibration & Error in beam parameter measurements & $\begin{array}{l}1.0 \% \text { in beam size error } \\
2.0 \% \text { in } 2 \mathrm{D} \text { emittance error } \\
2.9 \% \text { in intrinsic emittance error }\end{array}$ \\
\hline Cross-plane rotation of the profile monitor & Error in beam parameter measurements & $\begin{array}{l}0.5^{\circ} \text { in rotation error } \\
2.7 \% \text { in intrinsic emittance error }\end{array}$ \\
\hline Quadrupole field error & Error in transfer matrices & $\begin{array}{l}0.2 \% \text { of field error } \\
0.3 \% \text { in } 2 \mathrm{D} \text { emittance error } \\
1.0 \% \text { in intrinsic emittance error }\end{array}$ \\
\hline Quadrupole tilt & Additional coupling & $\begin{array}{l}1 \text { mrad of tilt } \\
\text { Negligible coupling contribution }\end{array}$ \\
\hline Energy uncertainty & $\begin{array}{l}\text { Error in transfer matrices and emittance } \\
\text { normalization }\end{array}$ & $\begin{array}{l}0.1 \% \text { in energy error } \\
0.3 \% \text { in } 2 \mathrm{D} \text { emittance error } \\
0.3 \% \text { in intrinsic emittance error }\end{array}$ \\
\hline Optics mismatch & Nonoptimal phase-space coverage & $\begin{array}{l}\text { Increase of statistical errors for } \\
\text { mismatched beams }\end{array}$ \\
\hline Chromaticity & Error in beam parameter measurements & Negligible \\
\hline Energy spread and dispersion & Error in beam parameter measurements & Negligible \\
\hline
\end{tabular}

indicate that the quadrupole field error is about $0.2 \%$. Simulations done with 10000 random seeds show that this converts to about $0.3 \%$ error in the apparent emittances and less than $1 \%$ error in the intrinsic emittances. The effect of field hysteresis can potentially also distort the measurement. In our case, however, experimental tests have proven that this effect is negligible-we have observed no significant differences between measurements for which the quadrupoles were cycled at every scan step and measurements for which the magnets were not cycled. This is attributed to the fact that the quadrupoles are weakly excited during the measurement because of the relatively low quadrupole strengths required for the measurement and relatively low beam energy in our case.

(vii) Quadrupole magnets may be rotated about their longitudinal axes, which would add an extra coupling to the beam. Quadrupole roll angles at the SITF do not exceed $1 \mathrm{mrad}$, which according to our simulations do not generate any significant additional coupling. In practice our measurement setup is coupling free.

(viii) An energy error is equivalent to having a systematic field error in all quadrupoles. In addition, an energy error also contributes to the error of the normalized emittances. According to the accuracy of beam momentum at the SITF, the energy error is about $0.1 \%$. Monte Carlo simulations done with 10000 seeds show that this translates into a systematic error of about $0.3 \%$ in the normalized apparent and intrinsic emittances.

(ix) If the initial Twiss parameters differ from the design ones, the actual phase-advance coverage will be somewhat different from the optimum one shown in Fig. 2. As a consequence, the error of the reconstructed 4D parameters will increase. In most of the situations, however, it will still be possible to obtain a solution to a first approximation. Then, some quadrupoles upstream of the measurement setup should be varied for matching the incoming optics to the design one. Figure 6 shows how the statistical error of the 2D horizontal emittance varies with the mismatch parameter, ${ }^{2}$ assuming that the relative beam size measurement error is $5 \%$. The figure clearly indicates that the error on the emittance evaluation increases with the mismatch parameter, hence the optics at the reconstruction point should always be matched to the nominal one to avoid a systematic increase of the errors of the reconstructed parameters. Even for strongly mismatched beams $(M \leq 4)$, however, the fit works most of the times and the emittance error is below $30 \%$. In practice it requires hardly more than two iterations to match the optics. The fact that we can easily match the optics gives us confidence in our optics model and the obtained results.

(x) If the beam has a momentum spread, chromatic effects can potentially add an error to the reconstructed parameters. In our SITF measurements, the energy spread is typically around $0.1 \%$. Simulation results show that chromatic effects are negligible even for the maximum energy spread expected for SwissFEL, namely, 1.4\%. More

\footnotetext{
${ }^{2}$ The mismatch parameter is defined as in [3]: $M=\frac{1}{2}\left(\beta \gamma_{d}-2 \alpha \alpha_{d}+\gamma \beta_{d}\right)$, where the subscript $d$ denotes the design values. For a perfectly matched beam $M=1$.
} 
detailed studies of chromatic effects in quadrupole scan measurements can be found in Ref. [19].

(xi) If the momentum spread is finite and the trajectory or dispersion is not zero at the location of the quadrupole magnets, some dispersion will be generated, which can distort the measurement results. At the SITF the trajectory deviation is of the order of a few hundred $\mu \mathrm{m}$ and dispersion is corrected down to about $1 \mathrm{~mm}$. Simulations show that with our typical energy spread dispersive effects are negligible. We have also simulated a more extreme case for a beam with an energy spread of $1.4 \%$ and an initial offset of $0.25 \mathrm{~mm}$ in both planes: the error in the $2 \mathrm{D}$ emittances is about $1 \%$ and the error in the intrinsic emittances is smaller than $4 \%$.

The effect of the systematic errors is summarized in Table I. The simulations done including simultaneously the sources of errors described above (except the initial trajectory offset) show that for this example the systematic and statistical errors of the 2D emittances are about $2.9 \%$ and $2.2 \%$, respectively, while the systematic and statistical errors of the intrinsic emittances are about $4.6 \%$ and $3.4 \%$, respectively. Equivalent error levels were obtained for different sets of incoming beam parameters. Owing to the proper control of the beam optics between point of measurement and point of reconstruction, the multiplequadrupole scan method proposed here is a flexible and robust tool for the reconstruction of the intrinsic emittances.

\section{Example of a measurement}

Figures 7 and 8 show an actual measurement at the SITF, with $10^{\circ}$ phase-advance steps. The errors shown in Fig. 7 correspond to the statistical uncertainties calculated from the statistical errors of the beam measurements. The beam size and $x-y$ correlation were measured 10 times in every measurement step. The beam energy was $230 \mathrm{MeV}$. The optics were well matched: the mismatch parameter (see footnote ${ }^{2}$ ) computed from the measurement was 1.06 in $x$ and 1.00 in $y$, causing no significant increase of the errors of the reconstructed parameters (see Fig. 6). The apparent and intrinsic normalized emittances as well as the coupling terms are indicated in Fig. 7. The emittance increase due to coupling was $5.4 \%(C=0.054)$ in this case.

\section{Coupling correction}

The beam coupling terms may be corrected by means of solenoidal and/or skew quadrupolar field. At the SITF two knobs are available, a quadrupole and a skew quadrupole placed in the gun solenoid magnet, designed for compensating solenoid field imperfections (Knob normal and Knob skew). However we noted that two more knobs could be created by resorting to the particular SITF solenoid configuration. The first two S-band structures are surrounded by four focusing solenoid magnets as shown in Fig. 9. In the nominal configuration the fields of the four
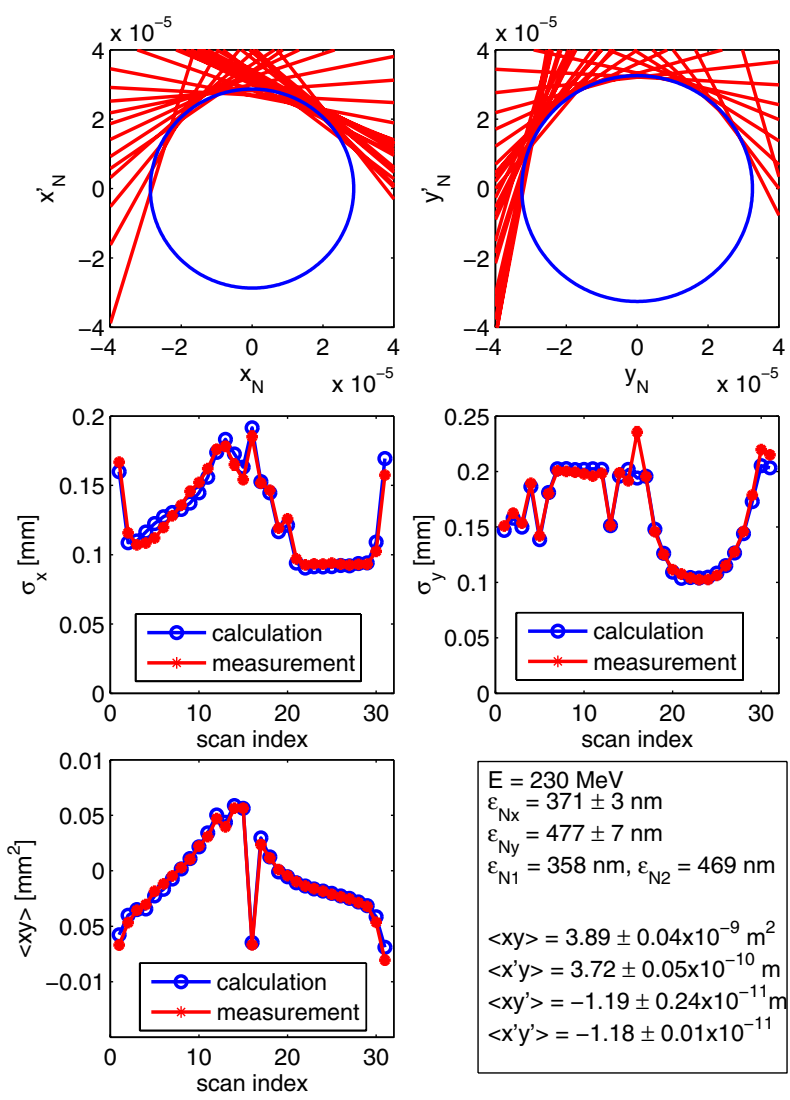

FIG. 7. Measurement example. The upper plots show the normalized phase space for $x$ (left) and $y$ (right) at the reconstruction point. The center and lower plots show the measured and calculated beam size and $x-y$ correlation terms at the measurement location. The reconstructed normalized apparent and intrinsic emittances as well as the coupling terms are indicated. The coupling increase due to transverse coupling is $5.4 \%$. See also Fig. 8.

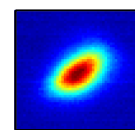

$$
1
$$

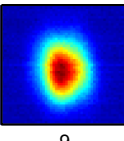

9

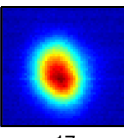

$\mathrm{y}[\mathrm{mm}]$
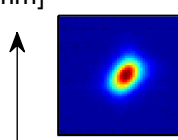

25

$\rightarrow \mathrm{x}[\mathrm{mm}]$

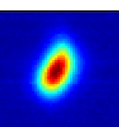

3

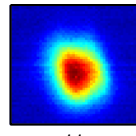

11

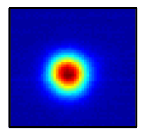

19

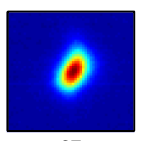

27

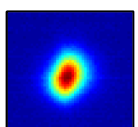

5

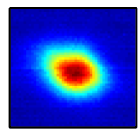

13

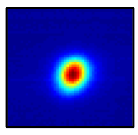

21

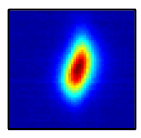

29

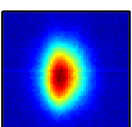

7

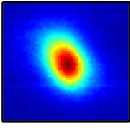

15

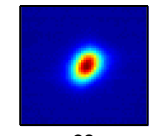

23

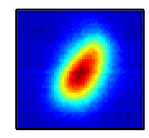

31
FIG. 8. Measurement example. Beam images for every other scan step. See also Fig. 7. 


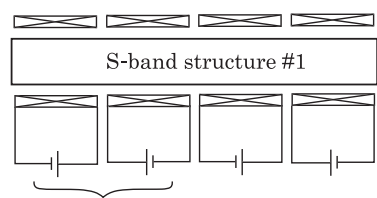

Knob-SB01

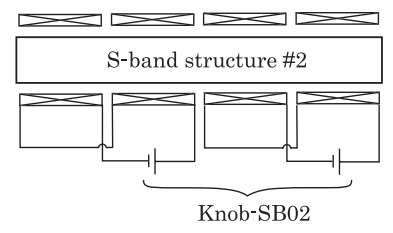

FIG. 9. S-band structures with solenoid magnets and their cabling (schematic). We use two pairs of power supplies (Knob$S B 01$ and Knob-SBO2) for the correction by applying asymmetric excitation, i.e., $I_{n} \pm I_{c}$, where $I_{n}$ is the nominal current and $I_{c}$ is the correction current.

solenoids of each structure are equal. By applying an asymmetric correction current at each couple of solenoids it is possible to vary the coupling correlations while maintaining approximately the same beam focusing (Knob-SBO1 and Knob-SBO2). The sensitivity matrix S, that is the effect of each knob on the four coupling terms at the point of interest, $s_{0}$, has been measured. It turned out that the solenoids of the second S-band structure, the Knob$S B 02$, are not very effective because of their cabling, which is not laid out for this purpose, and because of the higher beam energy at this position. The measured $\langle x y\rangle,\left\langle x y^{\prime}\right\rangle$, $\left\langle x^{\prime} y\right\rangle$, and $\left\langle x^{\prime} y^{\prime}\right\rangle$ at the point of interest are corrected
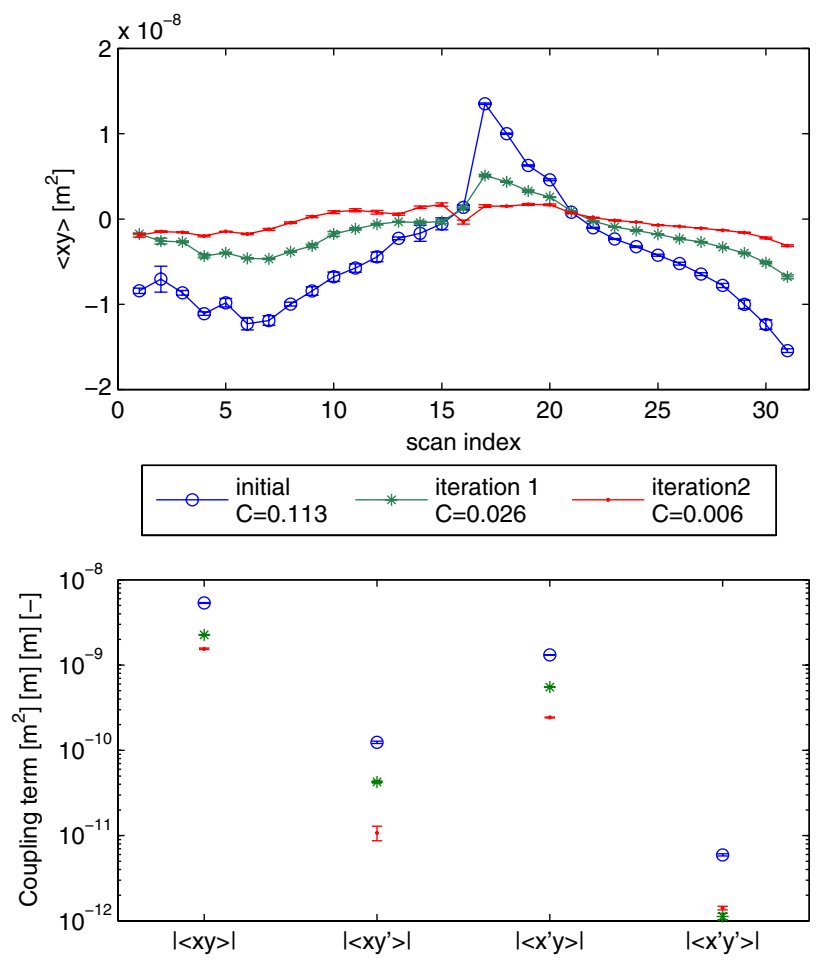

FIG. 10. Coupling correlations before and after the correction. The visible correlation term $\langle x y\rangle$ measured during the quadrupole scan (top). The four coupling correlations during the correction iteration (bottom). The initial coupling factor $C=0.113$ was reduced to $C=0.006$ after the correction procedure was repeated twice with a cut of $1 \%$. simply by applying the values of the four knobs strengths given by

$$
\vec{C}=S^{-1}\left(\begin{array}{c}
\langle x y\rangle \\
\left\langle x y^{\prime}\right\rangle \\
\left\langle x^{\prime} y\right\rangle \\
\left\langle x^{\prime} y^{\prime}\right\rangle
\end{array}\right) \text {. }
$$

Although the matrix is invertible, a matrix inversion with singular value decomposition (SVD) was employed to control the corrector knob strengths with a singular value cut. Without the cut we easily reach the limit of the knobs since the knob locations are not optimal. We note that the SVD matrix inversion is also applicable to cases where the matrix is not square.

After one iteration of the correction procedure, the coupling factor was largely suppressed (from 0.113 to 0.026); see Fig. 10. Because of the errors in the sensitivity matrix, a second correction iteration was required to reduce the coupling contribution to a negligible level $(C=0.006)$. A cut of $1 \%$ (i.e., singular values are cut if they are below $1 \%$ of the maximum) was applied in the matrix inversion. Without cut one of the knobs (Knob-SBO1) would have reached $98 \%$ of the available strength after the first iteration, while with this cut it reached only $14 \%$ of the maximum. For SwissFEL several dedicated skew quadrupoles will be installed to realize a set of efficient knobs, where the singular value cut may not be essential.

\section{CONCLUSION}

Four-dimensional measurements are needed to control the apparent emittance increase due to cross-plane coupling. We have presented an optimized procedure based on the multiple-quadrupole scan technique to measure the full 4D transverse beam matrix. This approach uses three quadrupole magnets and one profile monitor: the 10 second-order moments of the beam can be reconstructed from the beam sizes and the $x-y$ correlation measurements under different optics generated with the three quadrupoles. The method has been used for measuring the 4D beam parameters at the SITF. Simulations have shown that all 4D parameters can be reconstructed with small statistical and systematic errors-less than $3 \%$ in the 2D emittances and less than $5 \%$ in the intrinsic emittances. As the measurement is reliable and fast, beam coupling correction knobs could be calibrated by acquiring response matrices from the machine. The initial coupling factor could be reduced from 0.113 to 0.006 in two iterations.

\section{ACKNOWLEDGMENTS}

We thank Bolko Beutner, Sven Reiche, and Thomas Schietinger for fruitful discussions and careful proofreading of the manuscript. 
[1] C. Lejeune and J. Aubert, in Applied Charged Particle Optics, Part A. Advances in Electronics and Electron Physics, Supplement 13A, edited by A. Septier (Academic Press, New York, 1980).

[2] H. Wiedemann, Particle Accelerator Physics I (Springer, Berlin, 1998).

[3] M. Minty and F. Zimmermann, Measurement and Control of Charged Particle Beams (Springer, Berlin, 2003).

[4] F. Löhl, Master's thesis, University of Hamburg, 2005.

[5] F. Löhl, S. Schreiber, M. Castellano, G. Di Pirro, L. Catani, A. Cianchi, and K. Honkavaara, Phys. Rev. ST Accel. Beams 9, 092802 (2006).

[6] J. Bengtsson, W. Leemans, and T. Byrne, in Proceedings of the 15th Particle Accelerator Conference, Washington, DC, 1993 (IEEE, Piscataway, NJ, 1993), p. 567.

[7] P. G. Tenenbaum, CLIC-Note No. 326, 1997.

[8] M. D. Woodley and P. E. Emma, in Proceedings of the 20th International Linear Accelerator Conference, Monterey, CA, 2000 (SLAC, Menlo Park, CA, 2000), p. 196.

[9] P. Raimondi, P. J. Emma, N. Toge, N. J. Walker, and V. Ziemann, in Proceedings of the 15th Particle Accelerator
Conference, Washington, DC, 1993 (IEEE, Piscataway, NJ, 1993), p. 98.

[10] P. Tenenbaum et al., in Proceedings of the 16th Particle Accelerator Conference, Dallas, TX, 1995 (IEEE, New York, 1995), p. 749.

[11] H. Loos, in Proceedings of the 33rd International Free-Electron Laser Conference, Shanghai, 2011 (SINAP, Shanghai, 2012), p. 166.

[12] M. Pedrozzi et al., PSI Report No. 10-05, 2010.

[13] J. Buon, in Proceedings of the 15th Particle Accelerator Conference, Washington, DC, 1993 (IEEE, Piscataway, NJ, 1993), p. 469.

[14] K. Kubo, ATF Internal Report No. 99-02, 1999.

[15] P. Castro, DESY Technical-Note-2003-03, 2003.

[16] R. Ganter et al., PSI Report No. 10-04, 2012.

[17] M. Borland, APS LS Note No. 287, 2000.

[18] R. Ischebeck (private communication).

[19] A. Mostacci, M. Bellaveglia, E. Chiadroni, A. Cianchi, M. Ferrario, D. Filippetto, G. Gatti, and C. Ronsivalle, Phys. Rev. ST Accel. Beams 15, 082802 (2012). 